\title{
FINITE AND INFINITE SOLITON AND KINK-SOLITON TRAINS OF NONLINEAR SCHRÖDINGER EQUATIONS
}

\author{
STEFAN LE COZ AND TAI-PENG TSAI
}

\begin{abstract}
We will first review known results on multi-solitons of dispersive partial differential equations, which are special solutions behaving like the sum of many weakly-interacting solitary waves. We will then describe our recent joint work with Dong Li on nonlinear Schrödinger equations: Assuming the composing solitons have sufficiently large relative speeds, we prove the existence and uniqueness of a soliton train which is a multi-soliton composed of infinitely many solitons. In the $1 \mathrm{D}$ case, we can add to the infinite train an additional half-kink, which is a solution with a non-zero background at minus infinity.
\end{abstract}

\section{INTRODUCTION}

The nonlinear Schrödinger equation

$$
\left\{\begin{array}{l}
i u_{t}+\Delta u+f(u)=0, \\
u(t=0)=u_{0},
\end{array}\right.
$$

where $u$ is a complex valued function on $\mathbb{R} \times \mathbb{R}^{d}$ and the nonlinearity $f: \mathbb{C} \rightarrow \mathbb{C}$ is phase covariant, i.e. there exists $g: \mathbb{R}^{+} \rightarrow \mathbb{R}$ such that $f(z)=g\left(|z|^{2}\right) z$, appears in various physical contexts, for example in nonlinear optics or in the modelling of Bose-Einstein condensates. Mathematically speaking, it is one of the model nonlinear dispersive PDE, along with the Korteweg-De Vries equation and the nonlinear wave equation.

The local Cauchy theory in the energy space $H^{1}\left(\mathbb{R}^{d}\right)$ for $(\mathrm{NLS})$ is well understood (see e.g. [8] and the references cited therein). In this survey we are interested in the long time dynamics of global solutions. Essentially, two effects may be at play at large time. First of all, if the nonlinearity is not too strong, the linear part of the equation can dominate and solutions may behave as if they were solutions to the free linear Schrödinger equation. This is the scattering effect. On the other hand, in some cases the nonlinear term dominates and the solution tends to concentrate, with possible blow-up in finite time. This is the focusing effect. At the equilibrium between these two effects, one may encounter many different types of structures that neither scatter nor focus. The most common of these non-scattering global structures are the solitons, but there exist also dark solitons, kinks, etc. A

Date: April 19, 2016.

2010 Mathematics Subject Classification. 35Q55(35C08,35Q51).

Key words and phrases. soliton, multi-soliton, kink-soliton train, infinite soliton train, nonlinear Schrödinger equations.

The research of S. Le Coz is supported in part by the french ANR through project ESONSE. The research of Tsai is supported in part by NSERC grant 261356-13 (Canada). 
generic conjecture for nonlinear dispersive PDE is the Soliton Resolution Conjecture. Roughly speaking, it says that, as can be observed in physical settings, any global solution will eventually decompose at large time into a scattering part and well separated non-scattering structures, usually a sum of solitons. Apart from integrable cases (see e.g. 38]), such conjecture is usually out of reach (see nevertheless the recent breakthrough [17] on energy critical wave equation). Intermediate steps toward this conjecture are existence and stability results of configurations with well separated non-scattering structure, like multi-solitons, multi-kinks, infinite soliton and kink-soliton trains, etc. Our purpose in this paper is to review some of the existing results on this topic.

\section{SOlitons, DARK SOLITONS AND KINKS}

We review in this section some of the known elementary non-scattering structure solutions of NLS).

Let us first consider special solitons, the standing waves. A standing wave is a solution to (NLS) of the form $u(t, x)=e^{i \omega t} \phi(x)$, where $\omega \in \mathbb{R}$ and $0 \neq \phi \in H^{1}\left(\mathbb{R}^{d}\right)$ is a localized solution to the elliptic stationary equation

$$
-\Delta \phi+\omega \phi-f(\phi)=0 .
$$

Since the ground work of Berestycki and Lions [4, 5, it is well known that (1) admits solutions in $H^{1}\left(\mathbb{R}^{d}\right)$ if $\omega>0$ and $f(z)=g\left(|z|^{2}\right) z$ verify the following hypotheses.

Assumption 1. (energy-subcritical) Let $d \geq 1$. Suppose $f(z)=g\left(|z|^{2}\right) z$ with $g \in C^{0}([0, \infty), \mathbb{R}) \cap C^{2}((0, \infty), \mathbb{R}), g(0)=0$,

$$
\left|s g^{\prime}(s)\right|+\left|s^{2} g^{\prime \prime}(s)\right| \leq C \cdot\left(s^{\alpha_{1} / 2}+s^{\alpha_{2} / 2}\right), \quad \forall s>0,
$$

where $C>0,0<\alpha_{1} \leq \alpha_{2}<\alpha_{\max }, \alpha_{\max }=+\infty$ if $d=1,2, \alpha_{\max }=\frac{4}{d-2}$ if $d \geqslant 3$. (focusing) There exists $s_{0}>0$, such that

$$
G\left(s_{0}\right):=\int_{0}^{s_{0}} g(\tilde{s}) d \tilde{s}>\omega s_{0} .
$$

The profile solutions of $(1)$ in $H^{1}\left(\mathbb{R}^{d}\right)$ are in general called bound states. Among bound states, it is common to distinguish between the ground states and the excited states. Recall first that three quantities are conserved along the $H^{1}$-flow of (NLS): the energy, the mass and the momentum, defined as follows.

$$
\begin{gathered}
E(u):=\frac{1}{2}\|\nabla u\|_{2}^{2}-\int_{\mathbb{R}^{d}} F(u) d x, \quad F(z):=\int_{0}^{|z|} f(s) d s, \\
M(u):=\frac{1}{2}\|u\|_{2}^{2}, \quad P(u):=\Im \int_{\mathbb{R}^{d}} \bar{u} \nabla u d x .
\end{gathered}
$$

The ground states are minimizers of the action related to (1) (defined by $S=$ $E+\omega M$ ) and are in general positive, radial and unique (see [18, 23] and the recent progresses [7, 26]). For $d=1$, there exist only ground states, whereas for $d \geqslant 2$ there are infinitely many excited states.

A soliton is a standing wave of (NLS) that has been given a speed thanks to a Galilean transform. Since $(\mathrm{NLS})$ is Galilean invariant, a soliton is still a solution of NLS). Explicitly, a soliton with frequency $\omega>0$, speed $v \in \mathbb{R}^{d}$, initial phase $\gamma \in \mathbb{R}$ 
and position $x_{0} \in \mathbb{R}^{d}$ has the form

$$
R_{\phi, \omega, \gamma, x_{0}, v}:=\phi\left(x-v t-x_{0}\right) \exp \left(i\left(\frac{1}{2} v \cdot x-\frac{1}{4}|v|^{2} t+\omega t+\gamma\right)\right) .
$$

The dynamical properties of solitons are mostly known when the nonlinearity $f$ is of power-type $f(z)=|z|^{\alpha} z$ and the profile is a ground state (we will refer to such solitons as ground state solitons, opposed to excited state soliton or in general bound state soliton). For $L^{2}$-subcritical $\alpha\left(\alpha<\frac{4}{d}\right)$, the ground state soliton is orbitally stable 9] (i.e. stable up to phase shifts and translations), whereas for $L^{2}$-critical and supercritical $\alpha\left(\alpha=\frac{4}{d}, \alpha>\frac{4}{d}\right)$ it is unstable by blow-up [3, 37. (i.e. there exists an initial data in any neighborhood of the soliton such that the corresponding solution to (NLS) blows up in finite time). In general, excited state solitons are expected to be unstable [19, 21, 31, 32, 33.

For the focusing power-type nonlinearities, solitons are the only non-scattering solutions with a fixed profile, which is always localized. However, for other nonlinearities, there exist also other types of non-scattering solutions with a fixed profile, for example the so-called dark and grey solitons or the kinks.

A dark soliton is a travelling wave solution of (NLS) of the form $\phi(x-c t)$, where $c \in \mathbb{R}^{d}$ is the velocity and $\phi$ is a profile which has the particularity to be nonlocalized but with a constant modulus at infinity. The analysis of such type of solitons is much less developped than for localized (bright) solitons. Most of the works deal with the case where (NLS) is the Gross-Pitaevskii equation, i.e. when $f(z)=\left(1-|z|^{2}\right) z$. In this case, existence of dark solitons in dimension 1 follows from direct computations. One may refer to [10, 11] and the reference cited therein for a study of the existence and stability of dark solitons with generic nonlinearities in dimension 1. In higher dimension, existence of dark solitons has been a long time open problem. In the recent breakthrough [27, it has been proved that, for generic nonlinearities and in dimension $d \geqslant 3$, dark solitons exist for any speed between 0 and the speed of sound ( $\sqrt{2}$ in the case of the Gross-Pitaevskii nonlinearity).

A kink is a soliton-type solution to $(\mathrm{NLS})$ when $d=1$,

$$
K_{\phi_{K}, \omega, c, \gamma, x_{0}, v}:=\phi_{K}\left(x-(c+v) t-x_{0}\right) \exp \left(i\left(\frac{1}{2} v \cdot x-\frac{1}{4}|v|^{2} t+\omega t+\gamma\right)\right),
$$

but with a profile $\phi_{K}$ which has different limits at $-\infty$ and $+\infty$. Here $c$ is the intrinsic velocity associated to $\phi_{K}$. When $f$ is the Gross-Pitaevskii nonlinearity, i.e. $f(z)=\left(1-|z|^{2}\right) z$, there exists an explicit family of kink solutions (which are particular cases of dark solitons) given by $K(t, x)=\phi_{K}(x-c t)$,

$$
\phi_{K}(x)=\sqrt{\frac{2-c^{2}}{2}} \tanh \left(\frac{x \sqrt{2-c^{2}}}{2}\right)+i \frac{c}{\sqrt{2}}, \quad|c|<\sqrt{2} .
$$

In this paper, we will be particularly interested in cases where the kink profile $\phi$ has different limits with different modulus at $-\infty$ and $+\infty$. This will be the case if the nonlinearity $f$ verifies the following assumption.

Assumption 2. For some $\omega_{0}>0$, there is a first $b>0$ such that for $h(s)=$ $\omega_{0} s-f(s)$,

$$
h(b)=0, \quad h^{\prime}(b)>0, \quad \int_{0}^{b} h(s) d s=0 .
$$




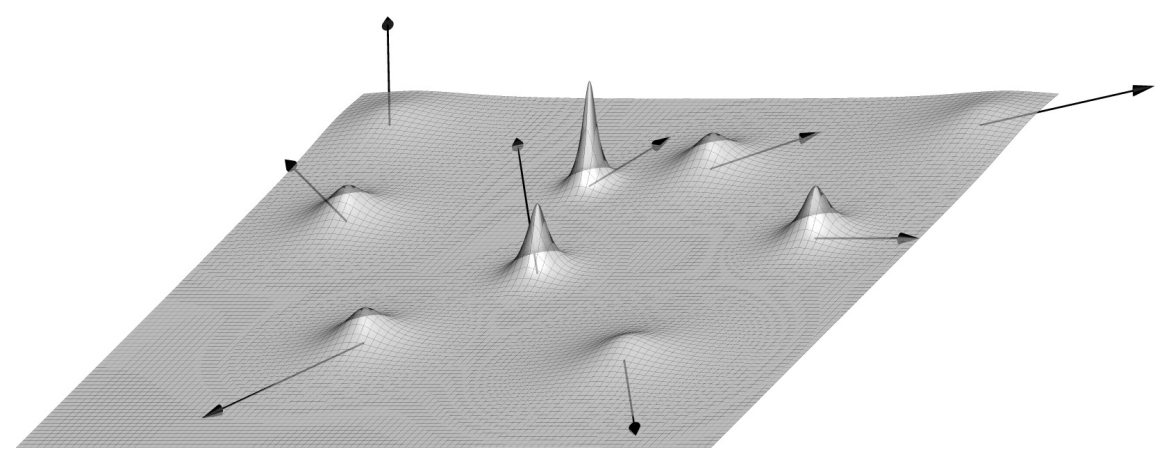

FigURE 1. Schematic representation of a multi-soliton

Under Assumption 2, there exists a (unique up to translation) kink-profile $\phi_{K} \in$ $\mathcal{C}^{2}(\mathbb{R})$ with zero intrinsic velocity such that

$$
\left\{\begin{array}{l}
-\phi_{K}^{\prime \prime}+\omega_{0} \phi_{K}-f\left(\phi_{K}\right)=0, \\
\lim _{x \rightarrow-\infty} \phi(x)=b, \quad \lim _{x \rightarrow+\infty} \phi(x)=0 .
\end{array}\right.
$$

To our knowledge, except in our works [24, 25], such kinks have never been investigated in the analysis of the long time behavior of solutions to (NLS). One reason for that is that they are genuinely infinite energy solutions and that no proper renormalization exists to make them energy finite.

\section{Multi-SOlitons, infinite SOliton trains And SOliton-Kink SOlutions}

As mentioned in Section 1 it is expected that global solutions to (NLS) will eventually decompose into a scattering part and well separated non-scattering structures. In this section, we review the existence and stability results for solutions composed of several of the elementary non-scattering structures described in Section 2. The basic example is the so-called multi-soliton, a solution of (NLS) build upon a finite number of solitons, Figure 1 contains a schematic representation of a multi-soliton. To be a little more precise, let

$$
R(t, x)=\sum_{j=1}^{N} R_{\phi_{j}, \omega_{j}, \gamma_{j}, x_{j}, v_{j}}(t, x)=: \sum_{j=1}^{N} R_{j}(t, x),
$$

where each $R_{j}$ is a soliton made from some parameters $\left(\omega_{j}, \gamma_{j}, x_{j}, v_{j}\right)$ and bound state $\phi_{j}$. As NLS is a nonlinear equation, $R$ is not a solution. What we call a multi-soliton is a solution $u$ of (NLS) such that

$$
\lim _{t \rightarrow+\infty}\|u-R\|_{X\left([t,+\infty) \times \mathbb{R}^{d}\right)}=0,
$$

where $X$ is some space-time function space, e.g. $L^{\infty}\left([0,+\infty), L^{2}\left(\mathbb{R}^{d}\right)\right)$.

3.1. The integrable cases. The first result of existence of multi-solitons was obtained in Zakharov and Shabat 38 in the case of the 1-d focusing cubic (i.e. $d=1$, $\left.f(z)=|z|^{2} z\right)$ nonlinear Schrödinger equation via the inverse scattering method. Indeed, in this particular case the equation is completely integrable and one can obtain multi-solitons in a rather explicit manner. The companion situation to the 1-d cubic case is the Gross-Pitaevskii case, i.e. $d=1$ and $f(z)=\left(1-|z|^{2}\right) z$. In 
that case (NLS) is again completely integrable, however no localized solitons exist. The counter parts of the solitons in this case are the dark solitons (2), which have modulus 1 at infinity. In this situation, it is also possible to show via the inverse scattering transform the existence of a solution of (NLS) behaving at large time as decoupled well-separated dark solitons. Note that in that case, due to the non-zero condition at infinity, the profile cannot be given simply as the sum of the dark solitons.

For the 1-d cubic (NLS), Kamvissis [22] showed that it is possible to push the inverse scattering analysis forward and obtain the existence of an infinite soliton train, i.e. a solution $u$ of (NLS) defined as in (4) but with $N=+\infty$ in the definition of $R$. In fact, the result given in 22 is sharper: it is shown that, if $d=1$, $f(z)=|z|^{2} z$ and under some technical hypotheses, any solution to (NLS) with initial data in the Schwartz class will eventually decompose at large time as an infinite soliton train and a "background radiation component". The existence of an infinite train of dark solitons has not been proved for the Gross-Pitaevskii case.

Other exotic solutions are available for 1-d cubic NLS, for example breather solutions which are periodic in space and whose magnitudes approach uniform values as $|t| \rightarrow \infty$, see e.g. [1].

3.2. Existence of multi-solitons, the energy method. Apart from the case previously mentioned, NLS is not completely integrable and it is generically not possible to apply the inverse scattering method to obtain the existence of multisolitons. The first existence result of multi-solitons in a non-integrable setting was obtained by Merle in 30 as a by-product of the proof of existence of multiple blowup points solutions for $L^{2}$-critical (NLS), i.e. $f(z)=|z|^{\frac{4}{d}} z$. The techniques initiated in 30 were then developed in [14, 15, 28, to obtain the following result.

Theorem 1 (Existence of multi-soliton by energy method, 14, 15, 28 ). Assume $f(z)=|z|^{\alpha} z$ with $0<\alpha<\alpha_{\max }$. Let $R$ be the profile given in (3). Let $\omega_{\star}$ and $v_{\star}$ be given by

$$
\omega_{\star}=\frac{1}{2} \min \left\{\omega_{j}, j=1, \ldots, N\right\}, \quad v_{\star}=\inf _{j, k=1, \ldots, N, j \neq k}\left|v_{j}-v_{k}\right| .
$$

There exist $\mu=\mu(d, N)$ and $v_{\sharp}:=v_{\sharp}\left(\phi_{1}, \ldots, \phi_{N}\right) \geqslant 0$ such that if $v_{\star}>v_{\sharp}$ then there exist $T_{0} \in \mathbb{R}$ and a solution $u \in \mathcal{C}\left(\left[T_{0},+\infty\right), H^{1}\left(\mathbb{R}^{d}\right)\right)$ of $(\mathrm{NLS})$ satisfying

$$
\|u(t)-R(t)\|_{H^{1}} \leqslant e^{-\mu \sqrt{\omega_{\star}} v_{\star} t}, \quad \forall t \geq T_{0} .
$$

If in addition all $\phi_{j}$ are ground states, then the result holds with $v_{\sharp}=0$, i.e., any $v_{*}>0$.

For the sake of simplicity, we have stated the result only for power-type nonlinearities, but its first part is in fact valid for any $\mathcal{C}^{1}$ nonlinearity verifying hypotheses a little stronger than Assumption 1. As mentioned before, the case $\alpha=\frac{4}{d}$ was treated by Merle [30, the ground state case by Martel and Merle [28] for $\alpha<\frac{4}{d}$ and Côte, Martel and Merle [15] for $\alpha>\frac{4}{d}$, and the excited state case by Côte and Le Coz [14.

The proofs in [14, 15, 28] follow a similar scheme. The idea is to choose an increasing sequence of time $\left(T^{n}\right)$ with $T^{n} \uparrow+\infty$ and consider the solutions $\left(u_{n}\right)$ to NLS which solve the equation backward in time with final data $u_{n}\left(T^{n}\right)=R\left(T^{n}\right)$. The sequence $\left(u_{n}\right)$ is an approximate sequence for a multi-soliton. To show its 
convergence, two arguments are at play. First, one shows that there exists a time $T_{0}$ independent of $n$ such that $u_{n}$ satisfies on $\left[T_{0}, T^{n}\right]$ the uniform estimates

$$
\left\|\left(u_{n}-R\right)(t)\right\|_{H^{1}} \leqslant e^{-\mu \sqrt{\omega_{\star}} v_{\star} t} .
$$

Second, we have compactness of the sequence of initial data $u_{n}\left(T_{0}\right)$, i.e. there exists $u_{0} \in H^{1}\left(\mathbb{R}^{d}\right)$ such

$$
u_{n} \rightarrow u_{0} \quad \text { strongly in } H^{s}\left(\mathbb{R}^{d}\right), \quad 0 \leqslant s<1 .
$$

To prove the uniform estimates, one first recall that the second derivative of the action around a soliton is coercive up to some $L^{2}$ scalar products. Precisely, given a soliton $R_{0}$ with parameters $\left(\omega_{0}, \gamma_{0}, x_{0}, v_{0}\right)$, the action is given by

$$
S=E+\left(\omega_{0}+\frac{\left|v_{0}\right|^{2}}{4}\right) M+v_{0} \cdot P
$$

and there exist $K \in \mathbb{N}$ and $\left(\xi_{k}^{0}\right)_{k=1, \ldots, K}$ such that we have for any $\varepsilon \in H^{1}\left(\mathbb{R}^{d}\right)$

$$
\left\langle S^{\prime \prime}\left(R_{0}\right) \varepsilon, \varepsilon\right\rangle \gtrsim\|\varepsilon\|_{H^{1}}^{2}-\sum_{k=1}^{K}\left|\left(\varepsilon, \xi_{k}^{0}\right)_{2}\right|^{2} .
$$

Hence to control the difference $\varepsilon=u_{n}-R$, it is enough to construct a functional $\mathcal{S}$ similar to $S$ but adapted to the multisoliton profile $R$ and to get rid of the bad $L^{2}$ scalar products. The construction of the functional is done by gluing together each functional $S_{j}$ suitably localized. The localization is possible since each bound state, henceforth each soliton, is exponentially decaying at infinity.

Getting rid of the bad $L^{2}$ scalar products is the trickiest part. In [28, the authors modulated the solitons in scaling, translation and phase to cancel the scalar products. This was possible only because they were in the $L^{2}$-subcritical case with ground states. In [15, the authors used a topological argument to select a final data which was not $R\left(T^{n}\right)$ but close enough to it, and such that for this final data the bad scalar products were vanishing. In [14, a bootstrap argument on the $L^{2}$ norm of $\varepsilon$ was used to get an a priori control of the type $\left|\left(\varepsilon, \xi_{k}^{0}\right)_{2}\right| \leqslant \frac{1}{v_{\star}}\|\varepsilon\|_{2}$, hence allowing to control the scalar products for $v_{\star}$ large enough.

The compactness argument is based on the virial identity and does not present major difficulty.

The energy method is very flexible and can be adapted to many other situations, e.g. for multi-solitons of Klein-Gordon equations [2, 16, or multi-speeds solitary waves of Schrödinger systems [20. It suits very well situations with a finite number of well localized solitons with finite energy. However, its implementation is far from being trivial when the number of solitons is infinite or when one soliton is replaced by a kink. In 24, 25, the authors have developped an approach suitable to situations where the energy technique fails to be directly applicable.

3.3. Existence of infinite trains and kink-solitons solutions, the fixed point argument. To prove the existence of solutions which have a priori infinite energy such as multi-solitons with kinks attached at both ends or infinite trains of solitons, an approach based on a fixed point argument around the desired profile has been followed in [24, 25]. 
We briefly recall the definition of the Strichartz space. For dimension $d \geq 1$, a pair of exponents $(q, r)$ is said (Schrödinger) admissible if

$$
\frac{2}{q}+\frac{d}{r}=\frac{d}{2}, \quad 2 \leq q, r \leq \infty, \text { and }(d, q, r) \neq(2,2, \infty) .
$$

On $I \times \mathbb{R}^{d}$ where $I \subset \mathbb{R}$, we define the Strichartz norm

$$
\|u\|_{S(I)}:=\sup _{(q, r) \text { admissible }}\|u\|_{L_{t}^{q} L_{x}^{r}\left(I \times \mathbb{R}^{d}\right)} .
$$

For $d=2$, we also need to impose $q>q_{1}$ in the above norm for some $q_{1}$ slightly larger than 2 to stay away from the forbidden endpoint. The Strichartz space $S(I)$ is the closure of all test functions in $\mathbb{R} \times \mathbb{R}^{d}$ under this norm. We denote by $N(I)$ the dual space of $S(I)$.

Let us first state a result on the existence of infinite soliton trains. Its conditions are far from optimal for simplicity of presentation. The same method can be applied to construct finite and infinite soliton trains for more general nonlinearities.

Theorem 2 (Existence of infinite soliton trains [24, 25]). Let $d \geq 1$. Assume $f(z)=|z|^{\alpha} z+g_{2}\left(|z|^{2}\right) z$ where $g_{2} \in C^{0}([0, \infty), \mathbb{R}) \cap C^{2}((0, \infty), \mathbb{R}), g(0)=0$,

$$
\left|s g_{2}^{\prime}(s)\right|+\left|s^{2} g_{2}^{\prime \prime}(s)\right| \leq C\left(s^{\alpha_{1.5} / 2}+s^{\alpha_{2} / 2}\right), \quad \forall s>0,
$$

where $C>0,0<\alpha<\alpha_{1.5} \leq \alpha_{2}<\alpha_{\max }$. There exist $r_{0}>\max \left(1, \frac{d \alpha}{2}\right), c_{1}>0$ and $v_{\sharp} \gg 1$ such that, if an infinite soliton train profile $R_{\infty}$ is given by

$$
R_{\infty}=\sum_{j=1}^{\infty} R_{j},
$$

with parameters $\omega_{j}>0, \gamma_{j} \in \mathbb{R}, x_{j}=0, v_{j} \in \mathbb{R}^{d}$ satisfying

- (uniform bound for bound states) for some $0<a<1$ and $C>0$,

$$
\left|\phi_{j}(x)\right|+\omega_{j}^{-1 / 2}\left|\nabla \phi_{j}(x)\right| \leq C \omega_{j}^{1 / \alpha} e^{-a \omega_{j}^{1 / 2}|x|}, \quad \forall x \in \mathbb{R}^{d}, \forall j \in \mathbb{N},
$$

- (Integrability)

$$
\sum_{j=1}^{\infty} \omega_{j}^{\frac{1}{\alpha}-\frac{d}{2 r_{0}}}<\infty
$$

- (High relative speeds)

$$
v_{*}=\inf _{j, k \in \mathbb{N}, j \neq k} \sqrt{\omega_{j}}\left|v_{k}-v_{j}\right| \geq v_{\sharp},
$$

- (Gradient bound)

$$
V_{*}=\sum_{j \in \mathbb{N}}\left\langle v_{j}\right\rangle \omega_{j}^{\frac{1}{\alpha}-\frac{d}{4}}<\infty \quad \text { if } \alpha<\frac{\alpha_{2}}{2+\alpha_{2}} .
$$

Then there exists a solution $u$ of NLS satisfying, for some $T_{0} \geq 0$,

$$
\left\|u-R_{\infty}\right\|_{S([t, \infty))} \leq e^{-c_{1} v_{\star} t}, \quad \forall t \geq T_{0} .
$$

Moreover, such profiles $R_{\infty}$ do exist for every such nonlinearity $f$.

Note that, in the integrable case, it was proved that an integrability condition on the parameters of the infinite trains is necessary for their existence, see 22, Remark $2]$.

We now switch to dimension $d=1$ to investigate the existence of solutions of NLS composed of kinks and solitons as represented in Figure 2 


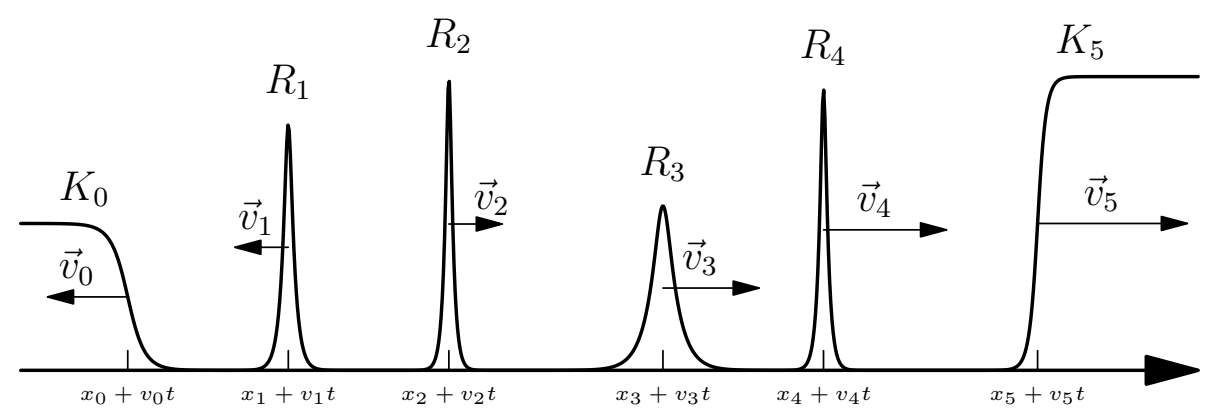

FigurE 2. Schematic representation of a kink-soliton train

Theorem 3 (Existence of finite kink-soliton trains [24]). Let $d=1$. Assume that $f$ is an energy subcritical nonlinearity such that there exists two kink profiles $\phi_{K_{0}}, \phi_{K_{N+1}}$ with $\phi_{K_{0}}^{\prime}$ and $\phi_{K_{N+1}}^{\prime}$ exponentially localized and the boundary conditions

$$
\begin{array}{ll}
\lim _{x \rightarrow-\infty} \phi_{K_{0}}(x) \neq 0, & \lim _{x \rightarrow+\infty} \phi_{K_{0}}(x)=0, \\
\lim _{x \rightarrow-\infty} \phi_{K_{N+1}}(x)=0, & \lim _{x \rightarrow+\infty} \phi_{K_{N+1}}(x) \neq 0 .
\end{array}
$$

Assume also the existence of $N$ soliton profiles $\phi_{1}, \ldots, \phi_{N}$. Define the profile

$$
W(t, x):=K_{0}(t, x)+\sum_{j=1}^{N} R_{j}(t, x)+K_{N+1}(t, x) .
$$

with parameters $\left(v_{j}, x_{j}, \omega_{j}, \gamma_{j}\right)_{j=0, \ldots, N+1} \subset \mathbb{R}^{4}$ such that $v_{0}<\cdots<v_{N+1}$. Define $v_{\star} b y$

$$
v_{\star}:=\inf \left\{\left|v_{j}-v_{k}\right| ; j, k=0, \ldots, N+1, j \neq k\right\} .
$$

Then there exist $v_{\sharp}>0$ (independent of $\left(v_{j}\right)$ ) large enough, $T_{0} \gg 1$ and constants $\mu_{1}, \mu_{2}>0$ such that if $v_{\star}>v_{\sharp}$, then there exists a (unique) multi-kink solution $u \in \mathcal{C}\left(\left[T_{0},+\infty\right), H_{\text {loc }}^{1}(\mathbb{R})\right)$ to NLS satisfying on $\left[T_{0},+\infty\right)$ the estimate

$$
e^{\mu_{1} v_{\star} t}\|u-W\|_{S([t,+\infty))}+e^{\mu_{2} v_{\star} t}\|\nabla(u-W)\|_{S([t,+\infty))} \leqslant 1 .
$$

In the above theorem, one kink can be dropped.

We may also have a kink attached to an infinite soliton train. For simplicity we choose a special nonlinearity. See [25] for general assumptions on $f$.

Theorem 4 (Existence of infinite kink-soliton trains [25]). Let $d=1$ and

$$
f(u)=|u|^{\alpha} u-|u|^{\beta} u, \quad 0<\alpha<\beta<\infty .
$$

It satisfies Assumption 2 and there is a kink profile $\phi_{K}$. If either $0<\alpha<4 / 3$, or $4 / 3 \leq \alpha<\sqrt{2}<\beta=2 / \alpha$, then there exist $r_{0}>1, c_{1}>0$ and $v_{\sharp} \gg 1$ such that, if an infinite kink-soliton train profile

$$
W=K+R_{\infty}=K+\sum_{j=1}^{\infty} R_{j}
$$

has its parameters satisfying the same assumptions as in Theorem 2 (with index $j$ starting from 0 ), then there exists a solution $u$ of (NLS) satisfying, for some $T_{0} \geq 0$,

$$
\|u-W\|_{S([t, \infty))} \leq e^{-c_{1} v_{\star} t}, \quad \forall t \geq T_{0} .
$$


Moreover, such profiles $W$ do exist.

The strategy for the proofs of Theorems 2 , 3 and 4 is the following. Let $W$ be a profile around which we want to build a solution. $W$ can be an infinite train, a kink-soliton train, etc. Since $W$ may be badly localized, we look for a solution of (NLS) in the form $u=W+\eta$, where $\eta$ satisfies the perturbation equation

$$
i \partial_{t} \eta+\Delta \eta+f(W+\eta)-f(W)+H=0,
$$

where $H$ is a source term coming from the fact that $W$ is not an exact solution, e.g. $H=f\left(R_{\infty}\right)-\sum_{j=1}^{+\infty} f\left(R_{j}\right)$ in the case of an infinite soliton train. In Duhamel formulation, the perturbation equation for $\eta$ becomes

$$
\eta(t)=-i \int_{t}^{\infty} e^{i(t-\tau) \Delta}(f(W+\eta)-f(W)+H) d \tau
$$

and the core of the proof is to perform a fixed point argument for this formulation. Here, two approaches are possible: One is based on a combination of the dispersive estimate

$$
\left\|e^{i t \Delta} u\right\|_{p} \lesssim|t|^{-d\left(\frac{1}{2}-\frac{1}{p}\right)}\|u\|_{\frac{p}{p-1}}, \quad \forall t \neq 0, \quad \forall p \in[2,+\infty],
$$

and Strichartz estimates

$$
\|u\|_{S\left(\left(t_{0}, \infty\right)\right)} \lesssim\left\|u_{0}\right\|_{2}+\|F\|_{N\left(\left(t_{0}, \infty\right)\right)} \quad \text { for } \quad i \partial_{t} u+\Delta u=F, \quad u\left(t_{0}\right)=u_{0} .
$$

The other uses only Strichartz estimates, but for both $u$ and $\nabla u$.

3.4. Open problems. We conclude this paper by reviewing some open problems related to the exotic solutions presented here.

3.4.1. Uniqueness. In Theorem 11 no uniqueness is proved for the multi-soliton. In Theorems 2, 3 and 4, the solution presented is unique in the class of solutions satisfying a strong decay estimate towards the desired profile; it does not preclude the possibility of existence of the same type of solution, but with a weaker decay towards the desired profile. In fact, it was proved in 14 that as soon as one of the composing soliton is linearly unstable, then there exists a one parameter family of multi-solitons converging toward the same profile. Hence in such cases uniqueness is not to be expected. However, one may hope that classification results hold, e.g. one may expect that for $L^{2}$-supercritical power-type nonlinearities, the multi-solitons converging toward a fixed $N$-sum of solitons form a $N$-parameter family. Such results were obtained for the Korteweg-de Vries equation by Combet 12. See also 13 for partial results in that direction for (NLS). For the kink-solitons solutions or the infinite trains, no result is available yet.

3.4.2. Stability. Another natural question coming to mind when investigating the exotic solutions of nonlinear Schrödinger equation is their stability. Again, the only available results concern finite multisolitons and the problem is completely open for infinite trains or kink-soliton solutions. For power-type nonlinearities, the only case where orbital stability of a multi-soliton holds is the Gross-Pitaevskii case, as has been proved by Béthuel, Gravejat and Smets for multi-dark-solitons in [6]. Stability of multi-solitons has been proved under restrictive hypotheses in 29] for orbital stability and in [34, 35, 36] for asymptotic stability. The hypotheses on the nonlinearity (e.g. high regularity or flatness at the origin) exclude in particular the power-type nonlinearities. 
3.4.3. Multikinks. One particular feature of the kinks considered in Theorem 3 is that they converge to 0 on one side. There exist however nonlinearities such that the kinks are connecting non-zero constants on both sides, for example -1 and 1 for the black solitons of Gross-Pitaevskii. There may also exist situations with kinks connecting e.g. 0 to 1 and kinks connecting 1 to 2 , etc. One would expect that it is possible to construct solutions by gluing together those kinks to get a solution with modulus having increasing terraces shape. Such solutions have never been constructed for nonlinear Schrödinger equation. One reason for that is the current lack of appropriate ansatz for such terraces shape solutions.

Many other related open problems exist, e.g. constructing solutions when one of the composing element is a line-soliton in 2 -d.

\section{REFERENCES}

[1] N. N. Akhmediev, V. M. Eleonskiŭ, and N. E. Kulagin. First-order exact solutions of the nonlinear Schrödinger equation. Teoret. Mat. Fiz., 72(2):183-196, 1987.

[2] J. Bellazzini, M. Ghimenti, and S. Le Coz. Multi-solitary waves for the nonlinear KleinGordon equation. Comm. Partial Differential Equations, 39(8):1479-1522, 2014.

[3] H. Berestycki and T. Cazenave. Instabilité des états stationnaires dans les équations de Schrödinger et de Klein-Gordon non linéaires. C. R. Acad. Sci. Paris Sér. I Math., 293(9):489-492, 1981.

[4] H. Berestycki and P.-L. Lions. Nonlinear scalar field equations I. Arch. Ration. Mech. Anal., 82:313-346, 1983.

[5] H. Berestycki and P.-L. Lions. Nonlinear scalar field equations II. Arch. Ration. Mech. Anal., 82(4):347-375, 1983

[6] F. Béthuel, P. Gravejat, and D. Smets. Stability in the energy space for chains of solitons of the one-dimensional Gross-Pitaevskii equation. Ann. Inst. Fourier (Grenoble), 64(1):19-70, 2014.

[7] J. Byeon, L. Jeanjean, and M. Mariş. Symmetry and monotonicity of least energy solutions. Calc. Var. Partial Differential Equations, 36(4):481-492, 2009.

[8] T. Cazenave. Semilinear Schrödinger equations. New York University - Courant Institute, New York, 2003.

[9] T. Cazenave and P.-L. Lions. Orbital stability of standing waves for some nonlinear Schrödinger equations. Comm. Math. Phys., 85(4):549-561, 1982.

[10] D. Chiron. Travelling waves for the nonlinear Schrödinger equation with general nonlinearity in dimension one. Nonlinearity, 25(3):813-850, 2012.

[11] D. Chiron. Stability and instability for subsonic traveling waves of the nonlinear Schrödinger equation in dimension one. Anal. PDE, 6(6):1327-1420, 2013.

[12] V. Combet. Multi-soliton solutions for the supercritical gKdV equations. Comm. Partial Differential Equations, 36(3):380-419, 2011.

[13] V. Combet. Multi-existence of multi-solitons for the supercritical nonlinear Schrödinger equation in one dimension. Discrete Contin. Dyn. Syst., 34(5):1961-1993, 2014.

[14] R. Côte and S. Le Coz. High-speed excited multi-solitons in nonlinear Schrödinger equations. J. Math. Pures Appl. (9), 96(2):135-166, 2011.

[15] R. Côte, Y. Martel, and F. Merle. Construction of multi-soliton solutions for the $L^{2}$ supercritical gKdV and NLS equations. Rev. Mat. Iberoam., 27(1):273-302, 2011.

[16] R. Côte and C. Muñoz. Multi-solitons for nonlinear Klein-Gordon equations. Forum Math. Sigma, 2:e15, 38, 2014.

[17] T. Duyckaerts, C. Kenig, and F. Merle. Classification of radial solutions of the focusing, energy-critical wave equation. Camb. J. Math., 1(1):75-144, 2013.

[18] B. Gidas, W. M. Ni, and L. Nirenberg. Symmetry of positive solutions of nonlinear elliptic equations in $\mathbf{R}^{n}$. In Mathematical analysis and applications, Part A, volume 7 of Adv. in Math. Suppl. Stud., pages 369-402. Academic Press, New York, 1981.

[19] M. Grillakis. Linearized instability for nonlinear Schrödinger and Klein-Gordon equations. Comm. Pure Appl. Math., 41(6):747-774, 1988. 
[20] I. Ianni and S. Le Coz. Multi-speed solitary wave solutions for nonlinear Schrödinger systems. J. Lond. Math. Soc. (2), 89(2):623-639, 2014.

[21] C. K. R. T. Jones. An instability mechanism for radially symmetric standing waves of a nonlinear Schrödinger equation. J. Differential Equations, 71(1):34-62, 1988.

[22] S. Kamvissis. Focusing nonlinear Schrödinger equation with infinitely many solitons. J. Math. Phys., 36(8):4175-4180, 1995.

[23] M. K. Kwong. Uniqueness of positive solutions of $\Delta u-u+u^{p}=0$ in $\mathbf{R}^{n}$. Arch. Rational Mech. Anal., 105(3):243-266, 1989.

[24] S. Le Coz, D. Li, and T.-P. Tsai. Fast-moving finite and infinite trains of solitons for nonlinear Schrödinger equations. Proc. Roy. Soc. Edinburgh Sect. A, 145(6):1251-1282, 2015.

[25] S. Le Coz and T.-P. Tsai. Infinite soliton and kink-soliton trains for nonlinear Schrödinger equations. Nonlinearity, 27(11):2689-2709, 2014

[26] M. Mariş. On the symmetry of minimizers. Arch. Ration. Mech. Anal., 192(2):311-330, 2009.

[27] M. Mariş. Traveling waves for nonlinear Schrödinger equations with nonzero conditions at infinity. Ann. of Math. (2), 178(1):107-182, 2013.

[28] Y. Martel and F. Merle. Multi solitary waves for nonlinear Schrödinger equations. Ann. Inst. H. Poincaré Anal. Non Linéaire, 23(6):849-864, 2006.

[29] Y. Martel, F. Merle, and T.-P. Tsai. Stability in $H^{1}$ of the sum of $K$ solitary waves for some nonlinear Schrödinger equations. Duke Math. J., 133(3):405-466, 2006.

[30] F. Merle. Construction of solutions with exactly $k$ blow-up points for the Schrödinger equation with critical nonlinearity. Comm. Math. Phys., 129(2):223-240, 1990.

[31] T. Mizumachi. Instability of bound states for 2D nonlinear Schrödinger equations. Discrete Contin. Dyn. Syst., 13(2):413-428, 2005.

[32] T. Mizumachi. Vortex solitons for 2D focusing nonlinear Schrödinger equation. Differential Integral Equations, 18(4):431-450, 2005.

[33] T. Mizumachi. Instability of vortex solitons for 2D focusing NLS. Adv. Differential Equations, 12(3):241-264, 2007.

[34] G. Perelman. Some results on the scattering of weakly interacting solitons for nonlinear Schrödinger equations. In Spectral theory, microlocal analysis, singular manifolds, volume 14 of Math. Top., pages 78-137. Akademie Verlag, Berlin, 1997.

[35] G. Perelman. Asymptotic stability of multi-soliton solutions for nonlinear Schrödinger equations. Comm. Partial Differential Equations, 29(7-8):1051-1095, 2004.

[36] I. Rodnianski, W. Schlag, and A. Soffer. Asymptotic stability of $N$-soliton states of nonlinear Schrödinger equations. 2003. arXiv:math/0309114.

[37] M. I. Weinstein. Nonlinear Schrödinger equations and sharp interpolation estimates. Comm. Math. Phys., 87(4):567-576, 1982/83.

[38] V. E. Zakharov and A. B. Shabat. Exact theory of two-dimensional self-focusing and onedimensional self-modulation of waves in nonlinear media. Šoviet Physics JETP, 34(1):62-69, 1972 .

Institut de Mathématiques De Toulouse,

Université Paul Sabatier

118 route de Narbonne, 31062 Toulouse Cedex 9

FRANCE

E-mail address: slecoz@math.univ-toulouse.fr

Department of Mathematics,

University of British Columbia,

VANCOUVER BC

CANADA V6T $1 \mathrm{Z} 2$

E-mail address: ttsai@math.ubc.ca 